\title{
Design of dynamically adjustable anamorphic fractional Fourier transformer
}

\author{
M. Fatih Erden ${ }^{a}$, Haldun M. Ozaktas ${ }^{a}$, Aysegul Sahin ${ }^{a}$, David Mendlovic ${ }^{b}$ \\ ${ }^{a}$ Electrical Engineering, Bilkent University, 06533 Bilkent, Ankara, Turkey \\ ${ }^{\mathrm{b}}$ Tel-Aviv University, Faculty of Engineering, 69978 Tel-Aviv, Israel
}

Received 25 June 1996; revised 18 September 1996; accepted 23 September 1996

\begin{abstract}
We form optical systems by using only free space portions and cylindrical lenses, and consider these systems as anamorphic fractional Fourier transformers. We dynamically adjust the transform order, scale factor and field curvature of both orthogonal dimensions of anamorphic fractional Fourier transformation by just changing the focal lengths of cylindrical lenses used in the proposed setups. Here, we also consider two approaches for implementing cylindrical lenses with dynamically adjustable focal lengths. There may also he some other methods to obtain cylindrical lenses having adjustable focal lengths which can successfully be used in these proposed setups.
\end{abstract}

\section{Introduction}

In this paper, we proposed centered optical systems composed of portions of free space with cylindrical lenses in between, under the standard approximations of Fourier optics [1], and considered these systems as anamorphic fractional Fourier transformers. We assume that, except for the focal lengths of the lenses, all the other parameters, such as the wavelength of input light and the lengths of free space sections, are fixed, so that we are able to dynanically adjust the parameters of anamorphic fractional Fourier transformation by changing only the focal lengths of the cylindrical lenses.

Up to this time, in a given setup which is proposed as fractional Fourier transformer, the desired fraction is obtained by playing not only with the focal lengths of lenses but also with the distances of free space portions. In this paper, we assume that only the focal lengths of cylindrical lenses are subject to changes. Thus, we are able to adjust the parameters of anamorphic fractional Fourier transformation in real timc without changing the optical setup configuration.

Both being dynamically adjustable and anamorphic, dynamically adjustable anamorphic fractional Fourier transformation can find its place in most of the applications. Some of these applications include time-variant or space-variant filtering, and signal detection [2-5], time-variant or space-variant multiplexing, and data compression [2], correlation, matched filtering, and pattern recognition [6,7], study of time-frequency or space-frequency distributions [2,8,9], and phase retrieval $[10,11]$.

The notion of introducing another degree of freedom to fractional Fourier transformation by use of anamorphic optics is presented in Ref. [12]. In this paper, we control the transform order, scale factor and the field curvature of both orthogonal dimensions (namely $x$ and $y$ ) of the anamorphic fractional Fourier transformation by just changing the focal lengths of the cylindrical lenses used in a given setup. With this motivation in mind, in Section 2 of this paper we defined anamorphic fractional Fourier transformation and related it to quadratic-phase systems. In Section 3, we proposed three optical setups. The first one is the simplest one but has limited control on the parameters of the anamorphic fractional Fourier transform. However, the third one has all the desired control on the parameters of the transform at the cost of increased complexity. Lastly, in Section 4, two approaches for implementing the cylindrical lenses are considered. In both methods, the focal length of the lenses is dynamically adjusted using hybrid opto-electronic deviccs. The first method is based on a liquid crystal active lens while the second one uses acoustooptic interaction in crystals. There may also be some other methods to obtain cylindrical lenses having adjustable focal lengths and suitable for our optical systems. 


\section{Anamorphic fractional Fourier transformation}

\subsection{Definition}

The ath order fractional Fourier transform $\left(\mathscr{F}^{a} \hat{q}\right)(u)$ of the function $\hat{q}(u)$ is defined for $0<|a|<2$ as

$$
\begin{aligned}
& \left(\mathscr{F}^{a} \hat{q}\right)(u) \equiv \int_{-\infty}^{\infty} B_{a}\left(u, u^{\prime}\right) \hat{q}\left(u^{\prime}\right) \mathrm{d} u^{\prime}, \\
& B_{a}\left(u, u^{\prime}\right) \equiv \frac{\exp [-i(\pi \hat{\phi} / 4-\phi / 2)]}{|\sin \phi|^{1 / 2}} \exp \left[i \pi\left(u^{2} \cot \phi-2 u u^{\prime} \csc \phi+u^{2} \cot \phi\right)\right],
\end{aligned}
$$

where

$$
\phi \equiv a \pi / 2
$$

and $\hat{\phi}=\operatorname{sgn}(\sin \phi)$. The kernel is defined separately for $a=0$ and $a= \pm 2$ as $B_{0}\left(u, u^{\prime}\right) \equiv \delta\left(u-u^{\prime}\right)$ and $B_{ \pm 2}\left(u, u^{\prime}\right) \equiv \delta(u$ $\left.+u^{\prime}\right)$ respectively [13]. The definition is easily extended outside the interval $[-2,2]$ by noting that $\mathscr{F}^{4 j+a} \hat{q}=\mathscr{F}^{a} \hat{q}$ for any integer $j$. Both $u$ and $u^{\prime}$ are interpreted as dimensionless variables.

Some essential properties of the fractional Fourier transform are: (i) It is linear. (ii) The first order transform $(a=1)$

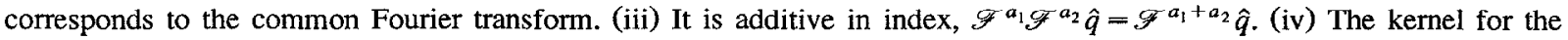
- ath order transform is the conjugate of the kernel for the ath order transform: $B_{a}^{*}\left(u, u^{\prime}\right)=B_{-a}\left(u, u^{\prime}\right)$. Other properties may be found in Refs. $[2,8,9,13-17]$.

By allowing for the possibility of a residual quadratic-phase term and a scale factor, the transformation kernel of fractional Fourier transformation in Eq. (1) can be generalized as

$$
h\left(x, x^{\prime}\right)=K \exp \left(i \pi x^{2} / \lambda R\right) \exp \left[\frac{i \pi}{s^{2}}\left(\frac{x^{2}}{M^{2}} \cot \phi-2 \frac{x x^{\prime}}{M} \csc \phi+x^{\prime 2} \cot \phi\right)\right],
$$

with $\phi=a \pi / 2$. This kernel maps a function $p(x / s)$ into $K^{\prime} \exp \left(i \pi x^{2} / \lambda R\right) p_{a}(x / s M)$, where $p_{a}(\cdot)$ is the ath order fractional Fourier transform of $p(\cdot)$, and $K^{\prime}$ is a new constant. In Eq. (3), $s$ is the unit in which $x$ and $x^{\prime}$ are measured, $M>0$ is referred to as the scale factor associated with the transformation and $R$ is the radius of the spherical surface on which the scaled fractional Fourier transform is observed. We call $\phi=a \pi / 2, M$ and $R$ as the parameters of the fractional Fourier transformation. We have determined the differential equations governing $\phi, M$ and $R$, and shown the relationship of these parameters to the parameters of a Gaussian beam in Ref. [18]. We see from Eq. (3) that the pure mathematical form of the fractional Fourier transformation in Eq. (1) is obtained when we set $s=1, M=1$ and $R=\infty$.

We can further generalize the fractional Fourier transformation definition to anamorphic fractional Fourier transformation definition by extending the kernel in Eq. (3) to two-dimensional systems, i.e.,

$$
\begin{aligned}
h\left(x, y, x^{\prime}, y^{\prime}\right)= & K \exp \left(i \pi x^{2} / \lambda R_{x}\right) \exp \left[\frac{i \pi}{s_{x}^{2}}\left(\frac{x^{2}}{M_{x}^{2}} \cot \phi_{x}-2 \frac{x x^{\prime}}{M_{x}} \csc \phi_{x}+x^{\prime 2} \cot \phi_{x}\right)\right] \\
& \times \exp \left(i \pi y^{2} / \lambda R_{y}\right) \exp \left[\frac{i \pi}{s_{y}^{2}}\left(\frac{y^{2}}{M_{y}^{2}} \cot \phi_{y}-2 \frac{y y^{\prime}}{M_{y}} \csc \phi_{y}+y^{\prime 2} \cot \phi_{y}\right)\right],
\end{aligned}
$$

with $\phi_{x}=a_{x} \pi / 2$ and $\phi_{y}=a_{y} \pi / 2$. As we see from this expression, $\phi_{x}, M_{x}$ and $R_{x}$ are the parameters of anamorphic fractional Fourier transformation along one orthogonal dimension (namely $x$ ), and $\phi_{y}, M_{y}$ and $R_{y}$ are the ones along the other dimension (namely $y$ ). Thus, a total anamorphic fractional Fourier transformation consists of six parameters.

\subsection{Relationship with quadratic-phase systems}

Thin lenses, arbitrary sections of free space (under the Fresnel approximation), quadratic graded-index media and any combinations of these belong to the class of quadratic-phase systems. In particular, the proposed centered optical systems that we consider in this paper also belong to this class. We characterize the members of quadratic-phase systems through linear transformation of the form [19-23]

$$
\begin{aligned}
& p_{\text {out }}(x)=\int_{-\infty}^{\infty} h\left(x, x^{\prime}\right) p_{\text {in }}\left(x^{\prime}\right) \mathrm{d} x^{\prime}, \\
& h\left(x, x^{\prime}\right)=K \exp \left[i \pi\left(\alpha x^{2}-2 \beta x x^{\prime}+\gamma x^{\prime 2}\right)\right],
\end{aligned}
$$


and generalize this transformation to two-dimensional systems as

$$
\begin{aligned}
& p_{\text {out }}(x, y)=\int_{-\infty}^{\infty} \int_{-\infty}^{\infty} h\left(x, y, x^{\prime}, y^{\prime}\right) p_{\text {in }}\left(x^{\prime}, y^{\prime}\right) \mathrm{d} x^{\prime} \mathrm{d} y^{\prime}, \\
& h\left(x, y, x^{\prime}, y^{\prime}\right)=K \exp \left[i \pi\left(\alpha_{x} x^{2}-2 \beta_{x} x x^{\prime}+\gamma_{x} x^{\prime 2}\right)\right] \exp \left[i \pi\left(\alpha_{y} y^{2}-2 \beta_{y} y y^{\prime}+\gamma_{y} y^{2}\right)\right],
\end{aligned}
$$

where $K$ is a complex constant, and $\alpha_{x}, \beta_{x}, \gamma_{x}, \alpha_{y}, \beta_{y}$ and $\gamma_{y}$ are real constants. We see from Eq. (6) that $K$ has no effect on the resulting spatial distribution, and as far as the spatial distributions of wave fields are concerned, a member of the class of two-dimensional quadratic-phase systems is completely specified by the six parameters $\alpha_{x}, \beta_{x}, \gamma_{x}, \alpha_{y}, \beta_{y}$ and $\gamma_{y}$.

An alternative way of completely specifying a member of the class of two-dimensional quadratic-phase systems is through its transformation matrix [19-23]

$$
\mathbf{T} \equiv\left[\begin{array}{cccc}
A_{x} & 0 & B_{x} & 0 \\
0 & A_{y} & 0 & B_{y} \\
C_{x} & 0 & D_{x} & 0 \\
0 & C_{y} & 0 & D_{y}
\end{array}\right] \equiv\left[\begin{array}{cccc}
\gamma_{x} / \beta_{x} & 0 & 1 / \beta_{x} & 0 \\
0 & \gamma_{y} / \beta_{y} & 0 & 1 / \beta_{y} \\
-\beta_{x}+\alpha_{x} \gamma_{x} / \beta_{x} & 0 & \alpha_{x} / \beta_{x} & 0 \\
0 & -\beta_{y}+\alpha_{y} \gamma_{y} / \beta_{y} & 0 & \alpha_{y} / \beta_{y}
\end{array}\right],
$$

with $A_{x} D_{x}-B_{x} C_{x}=1$ and $A_{y} D_{y}-B_{y} C_{y}=1$. If we know the kernel of a system, we can uniquely determine its transformation matrix or vice versa. There are some reasons for specifying the systems with their transformation matrices. If several systems each characterized by their transformation matrices are cascaded, the transformation matrix of the overall system can be found by multiplying the matrices of the cascaded systems $[19,21,22]$. Then, determination of the complete specification of the overall system boils down into elementary matrix multiplication. We can then uniquely determine the kernel of the linear transformation in Eq. (5) whenever we are interested in the transformation of wave fields. The transformation matrix defined above can also be viewed as the ray matrix employed in ray optical analysis $[1,20]$.

Specifically, the transformation kernel for a free-space propagation of length $d$ is expressed as

$$
h_{\text {free }}\left(x, y, x^{\prime}, y^{\prime}\right)=K_{\text {space }} \exp \left[i \pi\left(x-x^{\prime}\right)^{2} / \lambda d\right] \exp \left[i \pi\left(y-y^{\prime}\right)^{2} / \lambda d\right],
$$

and its corresponding transformation matrix is

$$
\mathbf{T}_{\text {free }}(d)=\left[\begin{array}{cccc}
1 & 0 & \lambda d & 0 \\
0 & 1 & 0 & \lambda d \\
0 & 0 & 1 & 0 \\
0 & 0 & 0 & 1
\end{array}\right] .
$$

Similarly, the kemel for a cylindrical lens with focal length $f_{x}$ along the $x$ direction is

$$
h_{x \text { lens }}\left(x, y, x^{\prime}, y^{\prime}\right)=K_{x \text { lens }} \delta\left(x-x^{\prime}\right) \exp \left(-i \pi x^{2} / \lambda f_{x}\right)
$$

with its transformation matrix

$$
\mathbf{T}_{x \text { iens }}\left(f_{x}\right)=\left[\begin{array}{cccc}
1 & 0 & 0 & 0 \\
0 & 1 & 0 & 0 \\
-1 / \lambda f_{x} & 0 & 1 & 0 \\
0 & 0 & 0 & 1
\end{array}\right]
$$

and the kernel for a cylindrical lens with focal length $f_{y}$ along the $y$ direction is

$$
h_{y \text { lens }}\left(x, y, x^{\prime}, y^{\prime}\right)=K_{y \text { lens }} \delta\left(y-y^{\prime}\right) \exp \left(-i \pi y^{2} / \lambda f_{y}\right)
$$

with its transformation matrix

$$
\mathbf{T}_{\text {ylens }}\left(f_{y}\right)=\left[\begin{array}{cccc}
1 & 0 & 0 & 0 \\
0 & 1 & 0 & 0 \\
0 & 0 & 1 & 0 \\
0 & -1 / \lambda f_{y} & 0 & 1
\end{array}\right]
$$

A large number of papers deal with the fractional Fourier transform in an optical context. For instance, see Refs. $[4,8,15-18,20,23-30]$ and the references therein. It is shown in Refs. $[18,20]$ that any quadratic-phase system can always be interpreted as a fractional Fourier transforming system. When we generalize this to two-dimensional systems, we say that any two-dimensional quadratic-phase system can always be interpreted as an anamorphic fractional Fourier transforming 
system. This fact is clearly seen when we compare the anamorphic fractional Fourier transformation kernel in Eq. (4) with the transformation kernel of the quadratic-phase systems in Eq. (6). We see from these expressions that the kernel in Eq. (4) is another form of the one in Eq. (6) with $\alpha_{x}=\cot \phi_{x} / s_{x}^{2} M_{x}^{2}+1 / \lambda R_{x}, \beta_{x}=\csc \phi_{x} / s_{x}^{2} M_{x}, \gamma_{x}=\cot \phi_{x} / s_{x}^{2}, \alpha_{y}=$ $\cot \phi_{y} / s_{y}^{2} M_{y}^{2}+1 / \lambda R_{y}, \beta_{y}=\csc \phi_{y} / s_{y}^{2} M_{y}$ and $\gamma_{y}=\cot \phi_{y} / s_{y}^{2}$. We can also find the transformation matrix associated with the anamorphic fractional Fourier transformation through Eq. (7) as

$$
\mathbf{T}=\left[\begin{array}{cc}
\overline{\mathbf{A}} & \overline{\mathbf{B}} \\
\overline{\mathbf{C}} & \overline{\mathbf{D}}
\end{array}\right]
$$

where

$$
\begin{aligned}
& \overline{\mathbf{A}}=\left[\begin{array}{cc}
M_{x} \cos \phi_{x} & 0 \\
0 & M_{y} \cos \phi_{y},
\end{array}\right], \\
& \overline{\mathbf{B}}=\left[\begin{array}{cc}
s_{x}^{2} M_{x} \sin \phi_{x} & 0 \\
0 & s_{y}^{2} M_{y} \sin \phi_{y},
\end{array}\right], \\
& \overline{\mathbf{C}}=\left[\begin{array}{cc}
-\sin \phi_{x} / s_{x}^{2} M_{x}+M_{x} \cos \phi_{x} / R_{x} & 0 \\
0 & -\sin \phi_{y} / s_{y}^{2} M_{y}+M_{y} \cos \phi_{y} / R_{y},
\end{array}\right]
\end{aligned}
$$

and

$$
\overline{\mathbf{D}}=\left[\begin{array}{cc}
\cos \phi_{x} / M_{x}+s_{x}^{2} M_{x} \sin \phi_{x} / \lambda R_{x} & 0 \\
0 & \cos \phi_{y} / M_{y}+s_{y}^{2} M_{y} \sin \phi_{y} / \lambda R_{y}
\end{array}\right]
$$

\section{The proposed optical setups}

The proposed setups are composed of sections of free space with cylindrical lenses in between. In the analysis of optical setups, we use the matrices in Eqs. (9), (11) and (13), and obtain the overall transformation matrix representation of the setup through ordinary matrix multiplication. After comparing it with the matrix in Eq. (14), we find the parameters of the anamorphic fractional Fourier transformation in terms of the optical setup parameters. We are able to obtain anamorphic fractional Fourier transformation by just inserting cylindrical lenses in optical setups. We assume that only the focal lengths of cylindrical lenses are subject to changes, so that we analyze the functional dependence of the parameters of anamorphic fractional Fourier transformation on the focal lengths of cylindrical lenses.

In these proposed optical setups, our primary objective is to change both $a_{x}$ and $a_{y}$ from -2 to 2 . In other words, we want to have any transform order we desire along each orthogonal dimensions of the anamorphic fractional Fourier transform. To achieve this, we propose the first optical setup which is the simplest one we can think of. In this setup we do not have any control on scale factors and field curvatures (i.e., $M_{x}, M_{y}, R_{x}$ and $R_{y}$ ). However, at each orthogonal dimension we may want to scale the fractional Fourier transform as we desire, and/or want to observe the fractional transform on a predefined curved surface. For this reason, we propose the second setup (which has some control on $M_{x}, M_{y}$, $R_{x}$ and $R_{y}$ ) and the third setup (which has all the desired control on $M_{x}, M_{y}, R_{x}$ and $R_{y}$ ) at the cost of increased complexity.

\subsection{Setup I}

We intend to obtain different fractional orders at different principal directions (namely $x$ and $y$ directions). As we can control only one direction with a single cylindrical lens, we need at least two cylindrical lenses corresponding to each direction. Then, the simplest optical setup that we can think of as an anamorphic fractional Fourier transformer is shown in Fig. 1. We get the transformation matrix $\mathbf{T}_{1}$ of the system through

$$
\mathbf{T}_{1}=\mathbf{T}_{\text {free }}\left(d_{2}\right) \mathbf{T}_{y \text { lens }}\left(f_{y}\right) \mathbf{T}_{x \text { lens }}\left(f_{x}\right) \mathbf{T}_{\text {free }}\left(d_{1}\right) .
$$




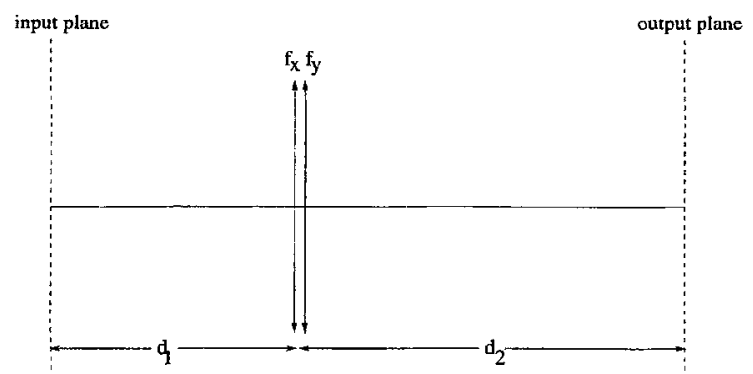

Fig. 1. The first proposed optical setup. Cylindrical lenses are denoted by solid arrows, and their focal length directions are indicated above them.

When we compare this matrix with the one in Eq. (14), we find the parameters of the anamorphic fractional Fourier transformation in terms of the optical setup parameters as

$$
\begin{aligned}
& \tan \phi_{x}=\frac{\lambda}{s_{x}^{2}} \frac{d_{1}+d_{2}-d_{1} d_{2} / f_{x}}{1-d_{2} / f_{x}}, \\
& \tan \phi_{y}=\frac{\lambda}{s_{y}^{2}} \frac{d_{1}+d_{2}-d_{1} d_{2} / f_{y}}{1-d_{2} / f_{y}}, \\
& M_{x}^{2}=\left(1-\frac{d_{2}}{f_{x}}\right)^{2}+\frac{\lambda^{2}}{s_{x}^{4}}\left(d_{1}+d_{2}-\frac{d_{1} d_{2}}{f_{x}}\right)^{2}, \\
& M_{y}^{2}=\left(1-\frac{d_{2}}{f_{y}}\right)^{2}+\frac{\lambda^{2}}{s_{y}^{4}}\left(d_{1}+d_{2}-\frac{d_{1} d_{2}}{f_{y}}\right)^{2}, \\
& \frac{1}{R_{x}}=\frac{\lambda}{s_{x}^{2}} \frac{\tan \phi_{x}}{M_{x}^{2}}-\frac{1}{f_{x}-d_{2}}, \\
& \frac{1}{R_{y}}=\frac{\lambda}{s_{y}^{2}} \frac{\tan \phi_{y}}{M_{y}^{2}}-\frac{1}{f_{y}-d_{2}} .
\end{aligned}
$$

We want to assign both to $a_{x}$ and $a_{y}$ any value between -2 and 2 by changing only the focal lengths $f_{x}$ and $f_{y}$ of the lenses. When we reexpress Eqs. (20) and (21) as

$$
\begin{aligned}
& f_{x}=\frac{d_{2}\left(s_{x}^{2} \tan \phi_{x}-\lambda d_{1}\right)}{s_{x}^{2} \tan \phi_{x}-\lambda\left(d_{1}+d_{2}\right)}, \\
& f_{y}=\frac{d_{2}\left(s_{y}^{2} \tan \phi_{y}-\lambda d_{1}\right)}{s_{y}^{2} \tan \phi_{y}-\lambda\left(d_{1}+d_{2}\right)},
\end{aligned}
$$

we can obtain the corresponding $f_{x}$ and $f_{y}$ values for each $\phi_{x}$ and $\phi_{y}$ values between $-\pi$ and $\pi$ (which corresponds to having $a_{x}$ and $a_{y}$, hetween -2 and 2). As a result, we can assign both to $a_{x}$ and $a_{y}$ any value between -2 and 2 by choosing $f_{x}$ and $f_{y}$ values appropriately. However, in this case, we do not have any control on $M_{x}, M_{y}, R_{x}$ and $R_{y}$. Their corresponding values for each $a_{x}$ and $a_{y}$ pair can be found from Eqs. (22)-(25).

\subsection{Setup II}

We may want to have some control on $M_{x}, M_{y}, R_{x}$ and $R_{y}$ while assigning to $a_{x}$ and $a_{y}$ any value between -2 and 2 . For this reason, we added two more cylindrical lenses to the setup in Fig. 1, and obtained the one in Fig. 2. We get the transformation matrix $\mathbf{T}_{2}$ of this system through

$$
\mathbf{T}_{2}=\mathbf{T}_{\text {free }}\left(d_{3}\right) \mathbf{T}_{y \text { lens }}\left(f_{y 2}\right) \mathbf{T}_{x \text { lens }}\left(f_{x 2}\right) \mathbf{T}_{\text {free }}\left(d_{2}\right) \mathbf{T}_{\text {ylens }}\left(f_{y 1}\right) \mathbf{T}_{x \text { lens }}\left(f_{x 1}\right) \mathbf{T}_{\text {free }}\left(d_{1}\right),
$$




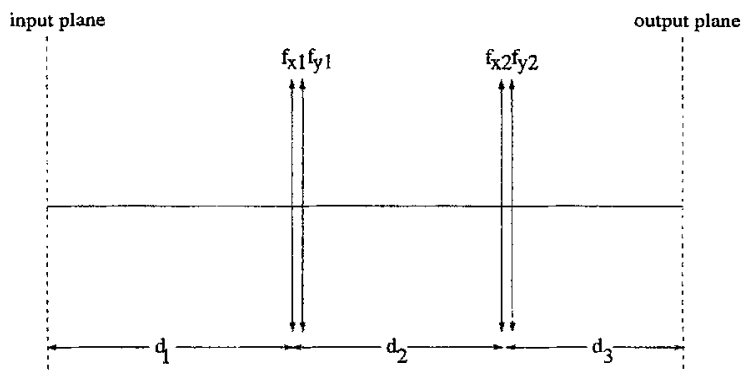

Fig. 2. The second proposed optical setup. Cylindrical lenses are denoted by solid arrows, and their focal length directions are indicated above them.

and compared this matrix with the one in Eq. (14). We find the parameters of the anamorphic fractional Fourier transformation in terms of the optical setup parameters as,

$$
\begin{aligned}
& \tan \phi_{x}=\frac{\lambda}{s_{x}^{2}} \frac{d_{1}\left(1-d_{3} / f_{x 2}\right)+\left(1-d_{1} / f_{x 1}\right)\left(d_{2}+d_{3}-d_{2} d_{3} / f_{x 2}\right)}{\left(1-d_{2} / f_{x 1}\right)\left(1-d_{3} / f_{x 2}\right)-d_{3} / f_{x 1}}, \\
& \tan \phi_{y}=\frac{\lambda}{s_{y}^{2}} \frac{d_{1}\left(1-d_{3} / f_{y 2}\right)+\left(1-d_{1} / f_{y 1}\right)\left(d_{2}+d_{3}-d_{2} d_{3} / f_{y 2}\right)}{\left(1-d_{2} / f_{y 1}\right)\left(1-d_{3} / f_{y 2}\right)-d_{3} / f_{y 1}}, \\
& M_{x}^{2}=\frac{\lambda^{2}}{s_{x}^{4}}\left[d_{1}\left(1-\frac{d_{3}}{f_{x 2}}\right)+\left(d_{2}+d_{3}-\frac{d_{2} d_{3}}{f_{x 2}}\right)\left(1-\frac{d_{1}}{f_{x 1}}\right)\right]^{2}+\left[\left(1-\frac{d_{2}}{f_{x 1}}\right)\left(1-\frac{d_{3}}{f_{x 2}}\right)-\frac{d_{3}}{f_{x 1}}\right]^{2}, \\
& M_{y}^{2}=\frac{\lambda^{2}}{s_{y}^{4}}\left[d_{1}\left(1-\frac{d_{3}}{f_{y 2}}\right)+\left(d_{2}+d_{3}-\frac{d_{2} d_{3}}{f_{y 2}}\right)\left(1-\frac{d_{1}}{f_{y 1}}\right)\right]^{2}+\left[\left(1-\frac{d_{2}}{f_{y 1}}\right)\left(1-\frac{d_{3}}{f_{y 2}}\right)-\frac{d_{3}}{f_{y 1}}\right]^{2}, \\
& \frac{1}{R_{x}}=\frac{\lambda}{s_{x}^{2}} \frac{\tan \phi_{x}}{M_{x}^{2}}-\frac{\left(1-d_{2} / f_{x 1}\right) / f_{x 2}+1 / f_{x 1}}{\left(1-d_{2} / f_{x 1}\right)\left(1-d_{3} / f_{x 2}\right)-d_{3} / f_{x 1}}, \\
& R_{y}=\frac{\lambda}{s_{y}^{2}} \frac{\tan \phi_{y}}{M_{y}^{2}}-\frac{\left(1-d_{2} / f_{y 1}\right) / f_{y 2}+1 / f_{y 1}}{\left(1-d_{2} / f_{y 1}\right)\left(1-d_{3} / f_{y 2}\right)-d_{3} / f_{y 1}} .
\end{aligned}
$$

This time, we are allowed to play with four parameters, namely the focal lengths $f_{x 1}, f_{y 1}, f_{x 2}$ and $f_{y 2}$ of the cylindrical lenses. Then, what we can achieve with the setup in Fig. 2 can be summarized as;

(i) We can assign to $a_{x}$ and $a_{y}$ any value between -2 and 2 , and can fix both $M_{x}$ and $M_{y}$ to a value $M$ which remains unchanged for all $a_{x}$ and $a_{y}$ pairs. However, in this case we do not have any control on $R_{x}$ and $R_{y}$.

(ii) Similarly, we can assign to $a_{x}$ and $a_{y}$ any value between -2 and 2 , and this time fix both $R_{x}$ and $R_{y}$ to a value $R$ which remains unchanged for all $a_{x}$ and $a_{y}$ pairs, at the cost of having no control on $M_{x}$ and $M_{y}$.

(iii) We again can assign to $a_{x}$ and $a_{y}$ any value between -2 and 2 , and this time satisfy both $M_{x}=M_{y}=M$ and $R_{x}=R_{y}=R$, but in this casc $M$ and $R$ takc different values for different $a_{x}$ and $a_{y}$ pairs.

\subsection{Setup III}

We may want to have all the desired control on $M_{x}, M_{y}, R_{x}$ and $R_{y}$ while assigning to $a_{x}$ and $a_{y}$ any value between -2 and 2. As the number of the parameters that we have to control is six, by adding two more cylindrical lenses with focal lengths $f_{x 3}$ and $f_{y 3}$ at the output plane of the setup in Fig. 2 (see Fig. 3), we can satisfy all the requirements on the parameters. To see this, let us concentrate on the previous setup. At the first item of the previous setup we said that, by playing with four focal lengths we can set $a_{x}, a_{y}, M_{x}$ and $M_{y}$ to any value we like. To be more concrete, for given $\phi_{x}=a_{x} \pi / 2, \phi_{y}=a_{y} \pi / 2, M_{x}$ and $M_{y}$, we find from Eqs. (29)-(32) that we have to choose the focal lengths as

$$
f_{x 1}=\frac{\left(s_{x}^{2} / \lambda\right) d_{2} M_{x} \sin \phi_{x}-d_{1} d_{2} M_{x} \cos \phi_{x}}{\left(d_{1}+d_{2}\right) M_{x} \cos \phi_{x}-\left(s_{x}^{2} / \lambda\right) M_{x} \sin \phi_{x}+d_{3}},
$$




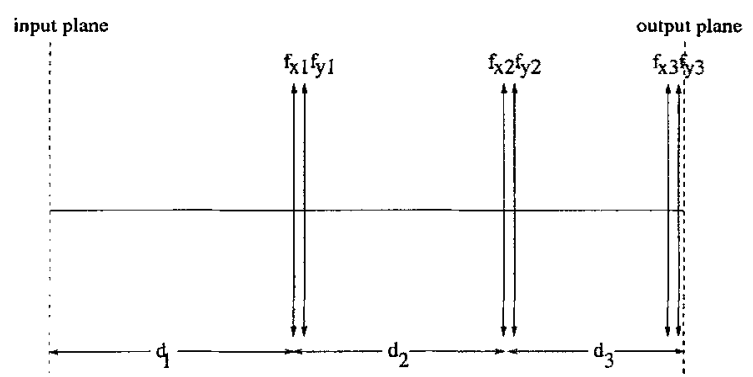

Fig. 3. The third proposed optical setup. Cylindrical lenses are denoted by solid arrows, and their focal length directions are indicated above them.

$$
\begin{aligned}
f_{y 1} & =\frac{\left(s_{y}^{2} / \lambda\right) d_{2} M_{y} \sin \phi_{y}-d_{1} d_{2} M_{y} \cos \phi_{y}}{\left(d_{1}+d_{2}\right) M_{y} \cos \phi_{y}-\left(s_{y}^{2} / \lambda\right) M_{y} \sin \phi_{y}+d_{3}}, \\
f_{x 2} & =\frac{d_{2} d_{3}}{d_{1} M_{x} \cos \phi_{x}-\left(s_{x}^{2} / \lambda\right) M_{x} \sin \phi_{x}+d_{2}+d_{3}},
\end{aligned}
$$

and

$$
f_{y 2}=\frac{d_{2} d_{3}}{d_{1} M_{y} \cos \phi_{y}-\left(s_{y}^{2} / \lambda\right) M_{y} \sin \phi_{y}+d_{2}+d_{3}} .
$$

In that case, we do not have any control on $R_{x}$ and $R_{y}$, and their corresponding values can be found from Eqs. (33) and (34). Now, looking at the setup in Fig. 3, with the help of the two lenses added at the end of the previous setup, we have the ability to set $R_{x}$ and $R_{y}$ to the desired ones $R_{d x}$ and $R_{d y}$ by choosing the focal lengths $f_{x 3}$ and $f_{y 3}$ as,

$$
1 / f_{x 3}=1 / R_{x}-1 / R_{d x}
$$

and

$$
1 / f_{y 3}=1 / R_{y}-1 / R_{d y} .
$$

Then, with the setup shown in Fig. 3, we can set all the parameters of anamorphic fractional Fourier transformer to their desired values by choosing the focal lengths satisfying the relations in Eqs. (35)-(40). In particular, when we dynamically adjust $f_{x 3}=R_{x}$ and $f_{y 3}=R_{y}$, we observe the anamorphic fractional Fourier transformation (with adjustable fraction and scale in both dimensions) of the input function on the planar surface at the output side.

\section{Implementations of a cylindrical lens with dynamically adjustable focal length}

In this section, the implementation aspects of the proposed system are discussed. The problematic device that determines the performance of the system is the dynamically adjustable focal length cylindrical lens.

Reconfigurable optical devices such as dynamically adjustable focal length lenses, are widely in use in various applications such as auto-focus and zoom-focus image capturing systems. Variable focus lenses can be implemented mechanically [31,32]. Several types of such lenses whose focal length can be controlled electronically have been proposed. Electrooptic effects are another option for modulating the refractive index distribution of a crystal [33]. Various types of variable focus lenses based on refractive index modulation of liquid crystals by an electric field have also been proposed [34-36].

With respect to the system proposed in this paper, we suggest to use a liquid crystal active lens for implementing the dynamically adjustable focal length leans. In this lens, a phase modulator is attached on one side of a conventional lens. The phase modulator should be a transmission type liquid crystal panel having a phase modulation capability of $2 \pi$ rad. The addressing of the panel is done electronically based on an electrode matrix. The number of electrodes in each direction is represented by $N$. When the phase modulator acts as a lens with a focal length $f$, it should imitate a phase distribution:

$$
\phi\left(x_{i}\right)=-\pi \frac{x_{i}^{2}}{\lambda f},
$$


when $x_{i}$ is the lateral location of pixel $i$ in the panel and $\lambda$ is the wavelength of the light. The phase difference of adjacent pixels is thus [36]:

$$
\Delta \phi\left(x_{i}\right)=\left|\phi\left(x_{i+1}\right)-\phi\left(x_{i}\right)\right| \approx 2 \pi a \frac{\left|x_{i}\right|}{\lambda f},
$$

where $a$ is the pitch of the panel.

A major problem of these panels is the reconfigurable rate that commonly is around the TV rate $(50 \mathrm{~Hz})$. In some cases, a much faster solution is needed. For those cases we look at an alternative solution that is faster but works in pulses. Here, we considered a method of simulating cylindrical lenses with dynamically adjustable focal lengths using the interaction of acoustic and optical waves within a medium. A closer look at acoustooptics shows that as far as the acoustic wave is concerned, the diffraction angle of the optical wave is only a function of the frequency of the acoustic wave when we fix acoustic wave amplitudes [37-40]. Then, at an instant of time, we can adjust the frequency distribution of the acoustic wave so that we can obtain the diffraction angle of the optical wave to be changing linearly with location in the optical aperture, which is equivalent to a cylindrical lens at that instant. In order to face with the index profile which simulates a cylindrical lens, we are forced to use pulse light. Here, we assume that the light wave with a small enough pulse width is much faster than the acoustic wave so that the acoustic wave, hence the refractive index profile, is assumed to be frozen during the pulse.

With this motivation in mind, our primary objective was to obtain a lens with a minimum achievable focal length so that we intend to have diffraction angles for the optical wave as large as possible. This necessitates the use of acoustic signals having larger bandwidths. This fact implies the use of birefringent devices because it is stated in Refs. [37-40] that one can obtain larger bandwidths by birefringent Bragg diffraction (when incident optical wave refractive index $n_{\mathrm{i}}$ is different from diffracted optical wave refractive index $n_{\mathrm{d}}$ ) compared to isotropic Bragg diffraction (when incident optical wave refractive index $n_{\mathrm{i}}$ is identical to diffracted optical wave refractive index $n_{\mathrm{d}}$ ). The complete explanation of birefringent Bragg diffraction requires consideration of the parametric interaction process [38].

We should note that the considerations of this section constitute theoretically motivated suggestions as to how lenses with dynamically variable focal lengths can be realized. However, the setups proposed in the previous section remain valid regardless of the method used to realize such lenses.

\section{Conclusion}

We know from Ref. [20] that any quadratic-phase system can always be interpreted as a fractional Fourier transforming system. As any combinations of thin lenses and arbitrary sections of free space are considered as quadratic phase systems, we proposed three optical systems which consisted of portions of free space with cylindrical lenses in between, and considered these systems as anamorphic fractional Fourier transformers. In these setups we achieved the transformers to be anamorphic by choosing the lenses to be cylindrical. In both of the proposed setups, rather than changing their system configurations completely, we were able to dynamically adjust the transform order of both orthogonal dimensions of anamorphic fractional Fourier transformation by just changing the focal lengths of the cylindrical lenses. The first setup was the simplest one, but other than the transform order, we could not have any control on the scale factor and the field curvature of both orthogonal dimensions of the transformation. However in the third setup, at the cost of increased complexity, we were able to adjust all the parameters (namely the transform order, scale factor and the field curvature) of both orthogonal dimensions by changing the focal lengths of the cylindrical lenses appropriately.

In the last section of this paper, we considered two methods of simulating a cylindrical lens with dynamically adjustable focal length. One of the methods is based on liquid crystal active lens while the other is based on acoustooptic interaction in crystals. However, in obtaining cylindrical lenscs with variable focal lengths, there may also be some other methods easier to implement, yet suitable for our proposed optical systems.

\section{Acknowledgements}

This work is partially supported by NATO's Scientific Affairs Division in the framework of the Science for Stability program.

\section{References}

[1] B.E.A. Saleh and M.C. Teich, Fundamentals of Photonics (Wiley, New York, 1991).

[2] H.M. Ozaktas, B. Barshan, D. Mendlovic and L. Onural, J. Opt. Soc. Am. A 11 (1994) 547. 
[3] M.A. Kutay, H.M. Ozaktas, O. Arikan and L. Onural, IEEE Trans. Signal Processing, submitted.

[4] M.F. Erden, H.M. Ozaktas and D. Mendlovic, Optics Comm. 125 (1996) 288.

[5] D. Mendlovic, Z. Zalevsky, A.W. Lohmann and R.G. Dorsch, Optics Comm. 126 (1996) 14.

[6] D. Mendlovic, H.M. Ozaktas and A.W. Lohmann, Appl. Optics 34 (1995) 303.

[7] D. Mendlovic, Y. Birran, R.G. Dorsch and A.W. Lohmann, Appl. Optics 12 (1995) 1665.

[8] A.W. Lohmann, J. Opt. Soc. Am. A 10 (1993) 2181.

[9] A.W. Lohmann and B.H. Soffer, J. Opt. Soc. Am. A 11 (1994) 1798.

[10] M.G. Raymer, M. Beck and D.F. McAlister, Phys. Rev. Lett. 72 (1994) 1137.

[11] D.F. McAlister, M. Beck, L. Clarke, A. Meyer and M.G. Raymer, Optics Lett. 20 (1995) 1181.

[12] D. Mendlovic, Y. Bitran, R.G. Dorsch, C. Ferreira, J. Garcia and H.M. Ozaktas, Appl. Optics 34 (1995) 745

[13] A.C. McBride and F.H. Kerr, IMA J. of Appl. Mathematics 39 (1987) 159.

[14] L.B. Almeida, IEEE Trans. on Signal Processing 42 (1994) 3084.

[15] H.M. Ozaktas and D. Mendlovic, Optics Comm. 101 (1993) 163.

[16] D. Mendlovic and H.M. Ozaktas, J. Opt. Soc. Am. A 10 (1993) 1875.

[17] H.M. Ozaktas and D. Mendlovic, J. Opt. Soc. Am. A 10 (1993) 2522.

[18] H.M. Ozaktas and M.F. Erden, J. Opt. Soc. Am. A, submitted.

[19] M.J. Bastiaans, J. Opt. Soc. Am. A 69 (1979) 1710.

[20] H.M. Ozaktas and D. Mendlovic, J. Opt. Soc. Am. A 12 (1995) 743.

[21] K.B. Wolf, Integral Transforms in Science and Engineering (Plenum Press, New York, 1979).

[22] M. Nazarathy and J. Shamir, J. Opt. Soc. Am. A 72 (1982) 356.

[23] S. Abe and J.T. Sheridan, Optics Lett. 19 (1994) 1801.

[24] A.W. Lohmann and D. Mendlovic, Appl. Optics 33 (1994) 7661.

[25] A.W. Lohmann, Optics Comm. 115 (1995) 437.

[26] L.M. Bernardo and O.D.D. Soares, J. Opt. Soc. Am. A 11 (1994) 2622.

[27] P. Pellat-Finet and G. Bonnet, Optics Comm. 111 (1994) 141.

[28] H.M. Ozaktas, B. Barshan and D. Mendlovic, Optical Review 1 (1994) 15.

[29] R.G. Dorsch, A.W. Lohmann, Y. Bitran, D. Mendlovic and H.M. Ozaktas, Appl. Optics 33 (1994) 7599.

[30] M.F. Erden, H.M. Ozaktas and D. Mendlovic, J. Opt. Soc. Am. A 13 (1996) 1068.

[31] J.M. Sasian, Opt. Eng. 29 (1990) 1181.

[32] B. Hulburd and D. Sandler, Opt. Eng. 29 (1990) 1186.

[33] T. Tatebayashi, T. Yamamoto and H. Sato, Appl. Optics 30 (1991) 3196.

[34] S.T. Kowel, P. Kornreich and A. Nouhi, Appl. Optics 23 (1984) 2774.

[35] P.F. Brinkley, S.T. Kowel and C. Chu, Appl. Optics 27 (1998) 4578.

[36] Y. Takaki and H. Ohzu, Optics Comm. 126 (1996) 123.

[37] M. Born and E. Wolf, Principles of Optics (Pergamon Press, 1975).

[38] J. Xu and R. Stroud, Acousto-Optic Devices (Wiley, New York, 1992).

[39] A. Vanderlugt, Optical Signal Processing (Wiley, New York, 1992).

[40] P.K. Das and C.M. DeCusatis, Acousto-Optic Signal Processing (Artech House, Inc., 1991). 